\title{
Health care provider experience with canagliflozin in real-world clinical practice: favorability, treatment patterns, and patient outcomes
}

This article was published in the following Dove Press journal:

International Journal of General Medicine

23 June 2017

Number of times this article has been viewed

\author{
Susan C Bolge' \\ Natalia M Flores ${ }^{2}$ \\ Shu Huang ${ }^{3}$ \\ Jennifer Cai'
}

'Janssen Scientific Affairs, LLC, Titusville, NJ, ${ }^{2}$ Kantar Health, Foster City, CA, ${ }^{3}$ Kantar Health, New York, NY, USA
Correspondence: Shu Huang Marketing Insights, Kantar Health, I I Madison Avenue, New York City, NY 10010, USA

Tel +I 2I27064263

Email shu.huang@kantarhealth.com
Purpose: This study describes how health care providers approach canagliflozin for the treatment of patients with type 2 diabetes mellitus (T2DM) in the real world.

Patients and methods: An Internet-based questionnaire was completed by 101 endocrinologists, 101 primary care physicians, and 100 nurse practitioners/physician assistants (NP/PAs). Health care providers were required to have experience prescribing or managing patients using canagliflozin to be included in the study. Health care providers compared canagliflozin with other T2DM medication classes on clinical characteristics, costs, and patient satisfaction. Confidence in canagliflozin was also measured. Health care providers reported their canagliflozin prescribing experience and good candidate characteristics for treatment. Finally, providers reported on patient outcomes among those receiving canagliflozin. All variables were compared across provider type. Results: Health care providers reported higher favorability for canagliflozin for blood pressure and body weight compared with dipeptidyl peptidase-4 (DPP-4) inhibitors and higher favorability for effect on blood pressure, body weight, treatment satisfaction, and glycosylated hemoglobin (HbA1c) compared with sulfonylureas (SUs), with differences observed for effect on blood pressure. Health care providers reported being very/extremely confident (55\%-74\%) with canagliflozin as a second- to fourth-line treatment. The top 3 characteristics reported by the providers, in terms of describing a good candidate for canagliflozin, include those concerned about their weight, insurance coverage/affordability, and avoiding injectable treatments. Finally, providers reported often/ always observing patients' lowering or controlling HbA1c (82\%-88\%) and improvement in overall quality of life (QoL; 50\%-53\%) with canagliflozin treatment. No differences were observed across provider type for confidence, good candidate characteristics, or patient outcomes.

Conclusion: Health care providers reported favorable experiences with canagliflozin and witnessed improvements in patients' clinical outcomes and QoL.

Keywords: diabetes, SGLT2-inhibitors, management goals, real-world evidence, prescribing experience

\section{Introduction}

Type 2 diabetes mellitus (T2DM) is a progressive and potentially debilitating chronic disease which can lead to kidney disease, retinopathy, neuropathy, and leg or foot ulcers in addition to increase risk for cardiovascular disease. T2DM is highly prevalent in the USA with recent estimates of $9.3 \%$ of the population having the condition ${ }^{1}$ and it is associated with a substantial economic and humanistic burden on society. ${ }^{2}$ To prevent the progression of T2DM and related complications, the most recent consensus guidelines of the American Diabetes Association (ADA) indicate that a concentration of glycosylated hemoglobin $(\mathrm{HbA} 1 \mathrm{c}) \geq 7.0 \%$ should serve as a trigger to initiate or 
escalate therapy. ${ }^{3}$ Notably, efforts to include treatment goals other than $\mathrm{HbA} 1 \mathrm{c}$ targets such as quality of care, health status of the patient, and costs have been emphasized by guidance to improve patient-centered care. ${ }^{3}$

Treatment escalation typically entails initiating therapy with oral monotherapy to adding more oral agents until a switch is made to insulin. ${ }^{3}$ In particular, the American Association of Clinical Endocrinologists (AACE) recommends the following suggested usage hierarchy for first-line monotherapy treatments when patients have $\mathrm{HbA} 1 \mathrm{c}<7.5 \%$ : metformin, glucagon-like peptide-1 (GLP-1) receptor agonist, sodium-glucose cotransporter (SGLT2) inhibitor, dipeptidyl peptidase-4 (DPP-4) inhibitor, or alpha-glucosidase inhibitor. ${ }^{4}$ Another oral treatment may be introduced in combination with a first-line oral agent if the HbAlc target is not reached within 3 months of monotherapy. According to AACE recommendations, caution should be taken with the addition of sulfonylurea/glinide (SU/GLN) or thiazolidinedione (TZD), with insulin in the combination treatment, due to the side effect profiles of SU/GLN and TZD, although ADA recommendations do not state this explicitly. ${ }^{3,4}$ In the case of a patient starting treatment with $\mathrm{HbA} 1 \mathrm{c} \geq 7.5 \%$, dual oral therapy is indicated as first-line treatment. If the treatment goals are not met after 3 months of dual therapy, another agent may be added, including insulin. If patients start treatment with $>9.0 \% \mathrm{HbA} 1 \mathrm{c}$, practitioners may use their discretion to start patients on dual or triple therapy and are advised to include insulin if there are diabetes-related symptoms present. ${ }^{4}$

Evidence suggests that oral agents' effects on body weight, risk of hypoglycemia, gastrointestinal side effects, systolic blood pressure, and heart rate may substantially differ between classes of oral agents. ${ }^{5}$ Metformin, DPP-4 inhibitors, GLP-1 agonists, and SGLT2 inhibitors have been found to reduce or maintain body weight, whereas SUs, TZDs, and insulin increase weight. SGLT2 inhibitors and GLP-1 agonists, in addition to metformin, have been found to reduce systolic blood pressure. There is a higher risk of cardiovascular events with SUs compared with metformin alone, and hypoglycemic events compared with most other treatments. Gastrointestinal side effects are more likely to occur with metformin than other agents, except GLP-1 agonists. SGLT2 inhibitors are associated with genital mycotic infections (GMIs). ${ }^{5}$

Canagliflozin, the first SGLT2 inhibitor to be approved for the treatment of T2DM in the USA in March 2013, is indicated as an adjunct to diet and exercise to improve glycemic control in adults with T2DM. ${ }^{6}$ By inhibiting SGLT2, canagliflozin leads to the inhibition of glucose reabsorption and increased urinary glucose excretion, thereby reducing blood glucose, body weight, and blood pressure. ${ }^{7}$ Canagliflozin $300 \mathrm{mg}$ also transiently blocks SGLT1 in the intestine, which reduces glucose absorption. ${ }^{8}$ In Phase III clinical trials, canagliflozin has been shown to improve HbA1c, body weight, and blood pressure, and to be generally well tolerated in patients with T2DM. ${ }^{7,9,10}$ Real-world studies have demonstrated similar improvements in $\mathrm{HbA} 1 \mathrm{c}$, body weight, and blood pressure among patients with T2DM using canagliflozin. ${ }^{11-14}$ Moreover, canagliflozin's unique mechanism has been suggested to be associated with weight loss and a low risk of hypoglycemia adding to its potential of benefiting health status and reducing costs. ${ }^{15}$

The current study seeks to understand how health care providers approached treatment with canagliflozin, assess perceptions of patient treatment experience with canagliflozin, and understand health care provider interactions with patients when discussing and initiating canagliflozin treatment.

\section{Materials and methods Sample and procedure}

US health care providers primary care physicians (PCPs; $\mathrm{n}=101)$, endocrinologists $(\mathrm{n}=101)$, and nurse practitioners/ physician assistants (NP/PAs; $n=100)$ were recruited into the survey. The sample for the study came from an online health care provider panel (Lightspeed All Global, part of Kantar Health), an actively managed double opt-in online panel where member registration and participation are maintained in the strictest confidence and are used purely for research purposes only. For this study, provider participants were targeted by specialty or type based on member registration profile. The physicians recruited for this study closely matched the demographics of the American Medical Association statistics with respect to age, gender, and region. Health care providers were invited to participate in this survey through an email invitation which contained the online survey link for self-administration from Lightspeed All Global or its panel partners. By clicking on the survey link, members were shown an informed consent form informing that participation is voluntary and responses will remain confidential detailing the research goals of the study. If agreed to, members will advance to the survey for completion. A convenience sample was used for health care provider recruitment; as such, no powering analysis was conducted. However, quotas were implemented to ensure close to equal representation of each provider type. Each 
eligible health care provider reported on their experiences with canagliflozin in a 15-minute Internet-based survey. This research study was submitted to the Pearl Institutional Review Board (Indianapolis, IN, USA) for review and was granted exemption status as this study involved survey/ interview procedures only, with no participant identifiers collected and no survey content that would have put the participants at risk should it be disclosed.

\section{Inclusion criteria}

To participate in the study, health care providers must have consented to participate in the study, should be board certified or be board eligible (if an endocrinologist or PCP), have at least 1 year of experience in practice, be a part of the decision-making process for prescribing (if NP/PA) or have written canagliflozin and/or canagliflozin-metformin prescriptions to patients with T2DM (if an endocrinologist or PCP), managing or treating at least 40 patients with T2DM, and managing or treating patients with TD2M who are receiving an SGLT2 inhibitor (specifically having managed at least 6 patients on canagliflozin and/or canagliflozin-metformin for at least 3 months).

\section{Measures}

The main grouping variable for this study was health care provider type, which had 3 groups: endocrinologists, PCPs, and NP/PAs. Health care providers reported on their primary specialty, sub-specialty, years in practice, gender, age, region of primary practice, practice specialty, and primary practice setting.

Health care providers were then asked what their perceptions of canagliflozin were when compared with other medication classes. Comparisons between canagliflozin and other medication classes for the treatment of T2DM (e.g., DPP-4s and SUs) were assessed on clinical characteristics, costs, and patient satisfaction. Confidence in canagliflozin's effect on various outcomes by line of therapy was also assessed. Health care providers reported on their current use of canagliflozin. Health care providers reported the proportion of patients who they prescribed SGLT2 inhibitors alone or in fixed-dose combination (FDC) with metformin or SGLT2 inhibitors (dapagliflozin, canagliflozin, empagliflozin, canagliflozinmetformin, dapagliflozin and metformin, empagliflozin and metformin for first through fourth lines). The health care provider's opinion on which characteristics identify a good patient candidate for canagliflozin, who makes the decision regarding treatments, and which attributes of canagliflozin are discussed, were also assessed.
Finally, providers reported on outcomes of patients on canagliflozin. Provider experience with canagliflozin on patient outcomes was assessed. In particular, the following were assessed: control of $\mathrm{HbA1c}$, control of blood pressure, weight loss, quality of life (QoL), emotional response to diabetes management, adherence to treatment, behavioral changes, and outlook on diabetes management.

\section{Analyses}

All analyses were carried out using SPSS version 20. All variables of interest were examined across provider type. Means and SDs were reported for continuous variables, and frequencies and percentages are reported for categorical variables. For categorical variables, chi-square tests were used to determine significant differences, whereas ANOVA was used for continuous variables when comparing provider types. An a priori null hypothesis threshold was set at $p<0.05$.

\section{Results}

\section{Health care provider characteristics}

A nationwide sample of providers was recruited into the survey. Of the total 302 completed surveys, 101 were endocrinologists, 101 were PCPs (of those 52.5\% were family medicine and $47.5 \%$ were internal medicine), and 100 were NP/PAs (of those $44 \%$ were NPs and $56 \%$ were PAs). NP/ PAs reported specializing in the following: diabetes (45.0\%), endocrinology (37.0\%), family/general health/primary care (64.0\%), and/or other (2.0\%) (Table 1). Refer Table S1 for practice patient characteristics.

\section{Perceptions of canagliflozin}

Health care providers reported higher favorability ratings of canagliflozin than DPP-4s on blood pressure and body weight and higher favorability ratings of canagliflozin than SUs on its effect on $\mathrm{HbA1c}$, blood pressure, body weight, risk of hypoglycemia, overall treatment safety, and patient satisfaction. There were no significant differences between favorability ratings among endocrinologists, PCPs, and NP/PAs regarding the effect of canagliflozin versus DPP-4s on HbA1c or canagliflozin versus SUs on HbA1c. Favorability ratings regarding the effect of canagliflozin versus DPP-4s on blood pressure, and canagliflozin versus SUs on blood pressure, were found to significantly differ among endocrinologists, PCPs, and NP/PAs ( $p=0.029$ and $p=0.001$, respectively), with endocrinologists having the most favorable view of canagliflozin. Although statistically different for canagliflozin versus SUs, favorability ratings were marginally different among endocrinologists, PCPs, and NP/PAs for effects on bodyweight for 
Table I Characteristics of providers and practices

\begin{tabular}{|c|c|c|c|c|c|c|c|c|c|}
\hline \multirow[t]{3}{*}{ Measures } & \multicolumn{8}{|c|}{ Provider type } & \multirow{3}{*}{$\begin{array}{l}\text { One-way } \\
\text { ANOVA } \\
\text { p-value }\end{array}$} \\
\hline & \multicolumn{2}{|c|}{$\begin{array}{l}\text { Endocrinologist } \\
(\mathrm{N}=10 \mathrm{I})\end{array}$} & \multicolumn{2}{|c|}{$P C P(N=101)$} & \multicolumn{2}{|c|}{ NP/PA $(N=100)$} & \multicolumn{2}{|c|}{ Total $(\mathbf{N}=302)$} & \\
\hline & Mean & SD & Mean & SD & Mean & SD & Mean & SD & \\
\hline \multirow[t]{2}{*}{ Years in practice } & $16.30^{\mathrm{a}}$ & 7.89 & $19.53^{b}$ & 6.87 & $13.72^{c}$ & 7.04 & 16.53 & 7.64 & $<0.001$ \\
\hline & $\mathbf{N}$ & $\%$ & $\mathbf{N}$ & $\%$ & $\mathbf{N}$ & $\%$ & $\mathbf{N}$ & $\%$ & $\begin{array}{l}\text { Chi-square } \\
\text { P-value or } \\
\text { Fisher's exact } \\
\text { test* }\end{array}$ \\
\hline \multicolumn{10}{|l|}{ Provider gender } \\
\hline Female & $19^{\mathrm{a}}$ & 18.8 & $22^{\mathrm{a}}$ & 21.8 & $74^{b}$ & 74.0 & 115 & 38.1 & $<0.001$ \\
\hline \multicolumn{10}{|l|}{ Provider age (years) } \\
\hline$<45$ & $36^{a}$ & 35.6 & $22^{\mathrm{b}}$ & 21.8 & $50^{c}$ & 50.0 & 108 & 35.8 & $<0.00 I^{*}$ \\
\hline $45-64$ & $54^{\mathrm{a}}$ & 53.5 & $7 I^{b}$ & 70.3 & $48^{\mathrm{a}}$ & 48.0 & 173 & 57.3 & \\
\hline$>65$ & $7^{\mathrm{a}}$ & 6.9 & $5^{a, b}$ & 5.0 & $\mathrm{I}^{\mathrm{b}}$ & 1.0 & 13 & 4.3 & \\
\hline Decline to answer & $4^{\mathrm{a}}$ & 4.0 & $3^{\mathrm{a}}$ & 3.0 & $\mathrm{I}^{\mathrm{a}}$ & 1.0 & 8 & 2.6 & \\
\hline \multicolumn{10}{|l|}{ Provider private practice setting } \\
\hline Private solo practice & $22^{\mathrm{a}, \mathrm{b}}$ & 21.8 & $30^{\mathrm{a}}$ & 29.7 & $15^{\mathrm{b}}$ & 15.0 & 67 & 22.2 & $<0.00 I^{*}$ \\
\hline Private group practice & $57^{\mathrm{a}}$ & 56.4 & $6 I^{\mathrm{a}}$ & 60.4 & $55^{\mathrm{a}}$ & 55.0 & 173 & 57.3 & \\
\hline Health system & $16^{\mathrm{a}}$ & 15.8 & $8^{a}$ & 7.9 & $13^{\mathrm{a}}$ & 13.0 & 37 & 12.3 & \\
\hline $\begin{array}{l}\text { Other (e.g., stand-alone hospital, } \\
\text { community center, or diabetes center) }\end{array}$ & $6^{a}$ & 5.9 & $2^{\mathrm{a}}$ & 2.0 & $17^{\mathrm{b}}$ & 17.0 & 25 & 8.3 & \\
\hline
\end{tabular}

Notes: *Test of independence conducted using Fisher's exact test when counts are $<5$. For pairwise comparisons, refer to superscripts (e.g., a, b, c) attached to column percentages. Column percentages in the same row that do not share the same superscripts (e.g., $X X^{a}$ vs $\left.X X^{b}\right)$ are significantly different at $p<0.05$. If the values share the same superscripts (e.g., $X X^{a}$ vs $X X^{a}$ or $X X^{a, b}$ vs $X X^{a}$ ) then they are not significantly different at $p<0.05$.

Abbreviations: ANOVA, analysis of variance; PCP, primary care physician; NP/PA, nurse practitioners/physician assistants.

canagliflozin versus DPP-4s, with endocrinologists favoring canagliflozin $(p=0.065)$. Favorability ratings on out-of-pocket costs for patients, risk of hypoglycemia, and patient satisfaction were not found to significantly differ among endocrinologists, PCPs, or NP/PAs (Table 2).

\section{Confidence by line of therapy}

More than $40 \%$ of endocrinologists and PCPs reported being very/extremely confident in canagliflozin as a first-line therapy, whereas $\sim 20 \%$ reported being moderately confident and $\sim 20 \%$ reported being slightly confident. The majority of NP/ PAs reported being slightly/moderately confident. Confidence ratings on canagliflozin as second-, third-, or fourth-line therapy did not significantly differ among provider types. Health care providers reported being very/extremely satisfied with canagliflozin for the second-line $(55 \%-59 \%)$, third-line $(60 \%-74 \%)$, and fourth-line (59\%-72\%) therapy (Table 3$)$.

\section{Current use of canagliflozin}

The proportion of patients who were prescribed SGLT2 inhibitors alone or in FDC with metformin was higher from first- to third-line therapy, with a slight decrease to fourth-line therapy. The proportion of patients who were prescribed SGLT2 inhibitors alone or in FDC with metformin who were prescribed canagliflozin was similar across first-line through fourth-line therapy, and did not statistically differ by provider type. The proportion of patients prescribed SGLT2 inhibitors alone or in FDC with metformin did not significantly differ among provider types for first- through fourth-line therapy (Table 4).

\section{Canagliflozin prescribing}

Health care providers were also asked to choose the top 3 characteristics from a list of 14 items (see full list in Table 5) that identify a patient as a good candidate for canagliflozin. Endocrinologists, PCPs, and NP/PAs each most often chose "overweight or have weight concerns", "insurance coverage/ affordability for long-term use", "good kidney function", "do not want an injectable/trying to delay injectables as long as possible", and "overall treatment safety", with no significant differences across provider types. Overall, 72\%-85\% of providers said that they made the final treatment decision often/always instead of their patients. This was found to differ among provider types with endocrinologists and PCPs reporting "often/always" making the final treatment decision for the patient $(85.1 \%)$, whereas fewer NP/PAs reported the same way $(71.7 \%)(p=0.016)$.

Various attributes of canagliflozin/canagliflozin-metformin treatment were discussed significantly less or more frequently 
Table 2 Canagliflozin/canagliflozin-metformin compared to DPP-4 inhibitor attributes for the treatment of T2DM by provider type

\begin{tabular}{|c|c|c|c|c|c|c|c|c|c|c|c|c|c|c|}
\hline \multirow[t]{4}{*}{ Measures } & \multicolumn{7}{|c|}{$\begin{array}{l}\text { Canagliflozin/canagliflozin-metformin } \\
\text { compared to DPP-4s }\end{array}$} & \multicolumn{7}{|c|}{$\begin{array}{l}\text { Canagliflozin/canagliflozin-metformin } \\
\text { compared to SUs }\end{array}$} \\
\hline & \multicolumn{6}{|c|}{ Provider type } & \multirow{3}{*}{$\begin{array}{l}\text { Chi- } \\
\text { square } \\
\text { p-value }\end{array}$} & \multicolumn{6}{|c|}{ Provider type } & \multirow{3}{*}{$\begin{array}{l}\text { Chi-square } \\
\text { p-value or } \\
\text { Fisher's } \\
\text { exact test* }\end{array}$} \\
\hline & \multicolumn{2}{|c|}{$\begin{array}{l}\text { Endocrinologist } \\
(\mathrm{N}=10 \mathrm{I})\end{array}$} & \multicolumn{2}{|c|}{$\begin{array}{l}P C P \\
(N=\mid 0 I)\end{array}$} & \multicolumn{2}{|c|}{$\begin{array}{l}\text { NP/PA } \\
(N=100)\end{array}$} & & \multicolumn{2}{|c|}{$\begin{array}{l}\text { Endocrinologist } \\
(N=100)\end{array}$} & \multicolumn{2}{|c|}{$\begin{array}{l}P C P \\
(N=101)\end{array}$} & \multicolumn{2}{|c|}{$\begin{array}{l}\text { NP/PA } \\
(N=101)\end{array}$} & \\
\hline & $\mathbf{N}$ & $\%$ & $\mathbf{N}$ & $\%$ & $\mathbf{N}$ & $\%$ & & $\mathbf{N}$ & $\%$ & $\mathbf{N}$ & $\%$ & $\mathbf{N}$ & $\%$ & \\
\hline \multicolumn{15}{|l|}{ Effect on HbAlc } \\
\hline More favorable & $45^{\mathrm{a}}$ & 44.6 & $26^{\mathrm{b}}$ & 25.7 & $38^{\mathrm{a}, \mathrm{b}}$ & 38.0 & 0.089 & 41 & 40.6 & 47 & 46.5 & 49 & 49.0 & $0.106 *$ \\
\hline Equally favorable & $39^{a}$ & 38.6 & $46^{\mathrm{a}}$ & 45.5 & $45^{\mathrm{a}}$ & 45.0 & & 34 & 33.7 & 24 & 23.8 & 36 & 36.0 & \\
\hline Less favorable & $12^{\mathrm{a}}$ & 11.9 & $23^{b}$ & 22.8 & $12^{\mathrm{a}}$ & 12.0 & & 23 & 22.8 & 24 & 23.8 & 11 & 11.0 & \\
\hline Do not know & $5^{a}$ & 5.0 & $6^{a}$ & 5.9 & $5^{a}$ & 5.0 & & 3 & 3.0 & 6 & 5.9 & 4 & 4.0 & \\
\hline \multicolumn{15}{|c|}{ Effect on blood pressure } \\
\hline More favorable & $64^{\mathrm{a}}$ & 63.4 & $40^{\mathrm{b}}$ & 39.6 & $49^{b}$ & 49.0 & 0.029 & $82^{\mathrm{a}}$ & 81.2 & $64^{\mathrm{b}}$ & 63.4 & $56^{b}$ & 56.0 & $0.001 *$ \\
\hline Equally favorable & $26^{a}$ & 25.7 & $37^{a}$ & 36.6 & $31^{a}$ & 31.0 & & $1 \mathrm{I}^{\mathrm{a}}$ & 10.9 & $28^{\mathrm{b}}$ & 27.7 & $24^{\mathrm{b}}$ & 24.0 & \\
\hline Less favorable & $6^{\mathrm{a}}$ & 5.9 & $16^{\mathrm{b}}$ & 15.8 & $10^{\mathrm{a}, \mathrm{b}}$ & 10.0 & & $6^{\mathrm{a}}$ & 5.9 & $5^{\mathrm{a}}$ & 5.0 & $9^{a}$ & 9.0 & \\
\hline Do not know & $5^{a}$ & 5.0 & $8^{\mathrm{a}}$ & 7.9 & $10^{\mathrm{a}}$ & 10.0 & & $2^{\mathrm{a}}$ & 2.0 & $4^{\mathrm{a}, \mathrm{b}}$ & 4.0 & $1 \mathrm{I}^{\mathrm{b}}$ & 11.0 & \\
\hline \multicolumn{15}{|c|}{ Effect on body weight } \\
\hline More favorable & $62^{\mathrm{a}}$ & 61.4 & $44^{b}$ & 43.6 & $52^{\mathrm{a}, \mathrm{b}}$ & 52.0 & 0.065 & 79 & 78.2 & 70 & 69.3 & 72 & 72.0 & $0.653^{*}$ \\
\hline Equally favorable & $22^{\mathrm{a}}$ & 21.8 & $35^{\mathrm{b}}$ & 34.7 & $34^{\mathrm{a}, \mathrm{b}}$ & 34.0 & & 11 & 10.9 & 18 & 17.8 & 17 & 17.0 & \\
\hline Less favorable & $10^{a, b}$ & 9.9 & $15^{\mathrm{a}}$ & 14.9 & $5^{\mathrm{b}}$ & 5.0 & & 8 & 7.9 & 11 & 10.9 & 7 & 7.0 & \\
\hline Do not know & $7^{\mathrm{a}}$ & 6.9 & $7^{\mathrm{a}}$ & 6.9 & $9^{a}$ & 9.0 & & 3 & 3.0 & 2 & 2.0 & 4 & 4.0 & \\
\hline \multicolumn{15}{|c|}{ Out-of-pocket cost for patient } \\
\hline More favorable & 20 & 19.8 & 18 & 17.8 & 30 & 30.0 & 0.339 & 28 & 27.7 & 16 & 15.8 & 19 & 19.0 & $0.164 *$ \\
\hline Equally favorable & 39 & 38.6 & 40 & 39.6 & 39 & 39.0 & & 11 & 10.9 & 21 & 20.8 & 19 & 19.0 & \\
\hline Less favorable & 29 & 28.7 & 26 & 25.7 & 19 & 19.0 & & 59 & 58.4 & 57 & 56.4 & 55 & 55.0 & \\
\hline Do not know & 13 & 12.9 & 17 & 16.8 & 12 & 12.0 & & 3 & 3.0 & 7 & 6.9 & 7 & 7.0 & \\
\hline \multicolumn{15}{|c|}{ Risk of hypoglycemia } \\
\hline More favorable & $12^{\mathrm{a}}$ & 11.9 & $18^{\mathrm{a}, \mathrm{b}}$ & 17.8 & $23^{b}$ & 23.0 & 0.064 & 76 & 75.2 & 63 & 62.4 & 63 & 63.0 & $0.23 I^{*}$ \\
\hline Equally favorable & $70^{\mathrm{a}}$ & 69.3 & $56^{b}$ & 55.4 & $46^{\mathrm{b}}$ & 46.0 & & 12 & 11.9 & 19 & 18.8 & 24 & 24.0 & \\
\hline Less favorable & $14^{a}$ & 13.9 & $18^{\mathrm{a}}$ & 17.8 & $23^{\mathrm{a}}$ & 23.0 & & 8 & 7.9 & 14 & 13.9 & 10 & 10.0 & \\
\hline Do not know & $5^{\mathrm{a}}$ & 5.0 & $9^{a}$ & 8.9 & $8^{a}$ & 8.0 & & 5 & 5.0 & 5 & 5.0 & 3 & 3.0 & \\
\hline \multicolumn{15}{|c|}{ Patient satisfaction } \\
\hline More favorable & 24 & 23.8 & 17 & 16.8 & 23 & 23.0 & 0.550 & 49 & 48.5 & 43 & 42.6 & 49 & 49.0 & 0.315 \\
\hline Equally favorable & 44 & 43.6 & 52 & 51.5 & 53 & 53.0 & & 31 & 30.7 & 40 & 39.6 & 40 & 40.0 & \\
\hline Less favorable & 25 & 24.8 & 21 & 20.8 & 16 & 16.0 & & 11 & 10.9 & 13 & 12.9 & 6 & 6.0 & \\
\hline Do not know & 8 & 7.9 & 11 & 10.9 & 8 & 8.0 & & 10 & 9.9 & 5 & 5.0 & 5 & 5.0 & \\
\hline
\end{tabular}

Notes: *Test of independence conducted using Fisher's exact test when counts are $<5$. For pairwise comparisons, refer to superscripts (e.g., a, b, c) attached to column percentages. Column percentages in the same row that do not share the same superscripts (e.g., $X X^{a}$ vs $\left.X X^{b}\right)$ are significantly different at $p<0.05$. If the values share the same superscripts (e.g., $X X^{a}$ vs $X X^{a}$ or $X X^{a, b}$ vs $X X^{a}$ ) then they are not significantly different at $p<0.05$.

Abbreviations: PCP, primary care physician; NP/PA, nurse practitioners/physician assistants; T2DM, type 2 diabetes mellitus; DPP4, dipeptidyl peptidase-4; SU, sulfonylureas; $\mathrm{HbAlc}$, glycosylated hemoglobin.

depending on provider type. These included: copay cards $(p=0.018)$, dosing and administration $(p=0.012)$, urge/need for frequent urination ( $p=0.015)$, impact on weight ( $p=0.007$ ), urinary tract infections (UTIs)/GMIs ( $p=0.017$ ), risk of hypoglycemia $(p=0.005)$, and overall treatment safety $(p=0.039)$. The attributes most frequently discussed with patients were $\mathrm{HbA} 1 \mathrm{c}$ reduction and potential for weight loss (Table 5).

\section{Canagliflozin outcomes}

Health care providers reported similar outcomes for their T2DM patients who were prescribed canagliflozin or FDC of canagliflozin with metformin. The majority of providers reported "often/always" observing patients' lowering or controlling $\mathrm{HbA} 1 \mathrm{c}$, weight loss, improvement in overall QoL, behaving positively by adhering to treatment and/or follow-up visits, and having a hopeful outlook for managing T2DM (Table 6).

\section{Discussion}

The Institute for Healthcare Improvement has developed the Triple Aim framework to optimize health care system performance. The Triple Aim framework calls for simultaneous pursuit of improvement in the health outcomes of populations, improvement in patients' experience of care, and reduction of per capita health care costs. ${ }^{16}$ Due to the high prevalence and chronic nature of T2DM, its comorbidity with other chronic conditions, and its numerous health complications, consideration of specific needs of patients with T2DM are necessary to reach the Triple Aim. In this study, health care providers reported experiencing improvements in 2 pillars 
Table 3 Confidence in canagliflozin/canagliflozin-metformin for each line of therapy

\begin{tabular}{|c|c|c|c|c|c|c|c|c|c|}
\hline \multirow[t]{3}{*}{ Line of Therapy } & \multicolumn{8}{|c|}{ Provider type } & \multirow{3}{*}{$\begin{array}{l}\text { Chi-square } p \text {-value } \\
\text { or Fisher's exact } \\
\text { test* }\end{array}$} \\
\hline & \multicolumn{2}{|c|}{$\begin{array}{l}\text { Endocrinologist } \\
(\mathrm{N}=10 \mathrm{I})\end{array}$} & \multicolumn{2}{|c|}{$P C P(N=|0|)$} & \multicolumn{2}{|c|}{ NP/PA $(N=100)$} & \multicolumn{2}{|c|}{ Total $(\mathbf{N}=302)$} & \\
\hline & $\mathbf{N}$ & $\%$ & $\mathbf{N}$ & $\%$ & $\mathbf{N}$ & $\%$ & $\overline{\mathbf{N}}$ & $\%$ & \\
\hline \multicolumn{10}{|l|}{ First line } \\
\hline Not at all confident & $24^{a}$ & 23.8 & $20^{\mathrm{a}, \mathrm{b}}$ & 19.8 & $13^{b}$ & 13.0 & 57 & 18.9 & 0.003 \\
\hline Slightly confident & $18^{\mathrm{a}}$ & 17.8 & $19^{a}$ & 18.8 & $33^{\mathrm{b}}$ & 33.0 & 70 & 23.2 & \\
\hline Moderately confident & $15^{\mathrm{a}}$ & 14.9 & $19 \mathrm{a}, \mathrm{b}$ & 18.8 & $28^{\mathrm{b}}$ & 28.0 & 62 & 20.5 & \\
\hline Very confident & $34^{a}$ & 33.7 & $25^{\mathrm{a}, \mathrm{b}}$ & 24.8 & $17^{\mathrm{b}}$ & 17.0 & 76 & 25.2 & \\
\hline Extremely confident & $10^{\mathrm{a}}$ & 9.9 & $18^{a}$ & 17.8 & $9^{a}$ & 9.0 & 37 & 12.3 & \\
\hline \multicolumn{10}{|l|}{ Second line } \\
\hline Not at all confident & $3^{\mathrm{a}}$ & 3.0 & $4^{a}$ & 4.0 & $4^{a}$ & 4.0 & 11 & 3.6 & $0.772^{*}$ \\
\hline Slightly confident & $14^{\mathrm{a}}$ & 13.9 & $1 I^{a}$ & 10.9 & $9^{a}$ & 9.0 & 34 & 11.3 & \\
\hline Moderately confident & $24^{a}$ & 23.8 & $30^{\mathrm{a}}$ & 29.7 & $3 I^{a}$ & 31.0 & 85 & 28.1 & \\
\hline Very confident & $42^{\mathrm{a}}$ & 41.6 & $32^{\mathrm{a}}$ & 31.7 & $38^{\mathrm{a}}$ & 38.0 & 112 & 37.1 & \\
\hline Extremely confident & $18^{\mathrm{a}}$ & 17.8 & $24^{\mathrm{a}}$ & 23.8 & $18^{\mathrm{a}}$ & 18.0 & 60 & 19.9 & \\
\hline \multicolumn{10}{|l|}{ Third line } \\
\hline Not at all confident & $\mathrm{I}^{\mathrm{a}}$ & 1.0 & $I^{a}$ & 1.0 & 0 & 0.0 & 2 & 0.7 & $0.149 *$ \\
\hline Slightly confident & $4^{a}$ & 4.0 & $8^{a}$ & 7.9 & $4^{a}$ & 4.0 & 16 & 5.3 & \\
\hline Moderately confident & $35^{a}$ & 34.7 & $18^{\mathrm{b}}$ & 17.8 & $22^{\mathrm{b}}$ & 22.0 & 75 & 24.8 & \\
\hline Very confident & $42^{\mathrm{a}}$ & 41.6 & $47^{\mathrm{a}}$ & 46.5 & $50^{\mathrm{a}}$ & 50.0 & 139 & 46.0 & \\
\hline Extremely confident & $19^{a}$ & 18.8 & $27^{a}$ & 26.7 & $24^{a}$ & 24.0 & 70 & 23.2 & \\
\hline \multicolumn{10}{|l|}{ Fourth line } \\
\hline Not at all confident & $3^{\mathrm{a}}$ & 3.0 & $3^{\mathrm{a}}$ & 3.0 & $\mathrm{I}^{\mathrm{a}}$ & 1.0 & 7 & 2.3 & $0.409 *$ \\
\hline Slightly confident & $9^{a}$ & 8.9 & $5^{a}$ & 5.0 & $10^{\mathrm{a}}$ & 10.0 & 24 & 7.9 & \\
\hline Moderately confident & $29^{a}$ & 28.7 & $22^{\mathrm{a}, \mathrm{b}}$ & 21.8 & $17^{\mathrm{b}}$ & 17.0 & 68 & 22.5 & \\
\hline Very confident & $40^{\mathrm{a}}$ & 39.6 & $44^{\mathrm{a}}$ & 43.6 & $43^{a}$ & 43.0 & 127 & 42.1 & \\
\hline Extremely confident & $20^{\mathrm{a}}$ & 19.8 & $27^{a}$ & 26.7 & $29^{a}$ & 29.0 & 76 & 25.2 & \\
\hline
\end{tabular}

Table 4 Prescribing by provider type for each line of therapy

\begin{tabular}{|c|c|c|c|c|c|c|}
\hline \multirow[t]{4}{*}{ Line of therapy } & \multicolumn{6}{|c|}{ Provider type } \\
\hline & \multirow{2}{*}{\multicolumn{2}{|c|}{$\begin{array}{l}\text { Endocrinologist } \\
(\mathrm{N}=10 \mathrm{I})\end{array}$}} & \multirow{2}{*}{\multicolumn{2}{|c|}{$\begin{array}{l}\text { PCP } \\
(N=10 I)\end{array}$}} & \multicolumn{2}{|c|}{ NP/PA $(N=100)$} \\
\hline & & & & & & \\
\hline & Mean & SD & Mean & SD & Mean & SD \\
\hline \multicolumn{7}{|l|}{ First line } \\
\hline \multirow[t]{2}{*}{ SGLT2 inhibitors alone or in FDC with metformin } & 5.04 & 7.61 & 5.8 & 7.21 & 5.6 & 7.96 \\
\hline & 41.47 & 23.1 & 41.72 & 22.2 & 46.03 & 21.1 \\
\hline \multicolumn{7}{|l|}{ Second line } \\
\hline \multirow[t]{2}{*}{ SGLT2 inhibitors alone or in FDC with metformin } & $|2.6|$ & 10.89 & 11.48 & 8.19 & 12.01 & 10.52 \\
\hline & 43.11 & 23.18 & 45.89 & 21.82 & 49.39 & 24.86 \\
\hline \multicolumn{7}{|l|}{ Third line } \\
\hline \multirow[t]{2}{*}{ SGLT2 inhibitors alone or in FDC with metformin } & 15.68 & 13.96 & 13.76 & 11.22 & 12.73 & 10.32 \\
\hline & 45.34 & 24.14 & 44.82 & 22.38 & 51.74 & 24.52 \\
\hline \multicolumn{7}{|l|}{ Fourth line } \\
\hline \multirow[t]{2}{*}{ SGLT2 inhibitors alone or in FDC with metformin } & 12.03 & 10.66 & 10.63 & 10.95 & 11.25 & 12.17 \\
\hline & 42.6 & 25.65 & 42.33 & 20.25 & 46.45 & 23.63 \\
\hline
\end{tabular}

Notes: Sample sizes for those who prescribed SLGT2 inhibitors for first line: endocrinologist ( $n=62), P C P(n=65)$, NP/PA ( $n=59)$; line 2: endocrinologist ( $n=88)$, PCP ( $n=87$ ), NP/PA ( $n=85)$; line 3: endocrinologist $(n=90)$, PCP $(n=87)$, NP/PA $(n=86)$; line 4: endocrinologist $(n=83)$, PCP ( $n=73)$, NP/PA ( $n=69)$. There were no statistically significant differences across provider type.

Abbreviations: PCP, primary care physician; NP/PA, nurse practitioners/physician assistants; FDC, fixed-dose combination.

of the Triple Aim framework, health outcomes and patients' experience of care, among patients using canagliflozin in real-world clinical practice.
Health care providers have witnessed positive clinical outcomes among patients using canagliflozin, including HbA1c lowering, weight loss, and blood pressure reduction. 
Table 5 Canagliflozin/canagliflozin-metformin prescribing

\begin{tabular}{|c|c|c|c|c|c|}
\hline & & \multicolumn{3}{|l|}{ Provider type } & \multirow{2}{*}{$\begin{array}{l}\text { Chi-square } \\
\text { p-value or } \\
\text { Fisher's } \\
\text { exact test* }\end{array}$} \\
\hline & & $\begin{array}{l}\text { Endocrinologist } \\
(\mathrm{N}=10 \mathrm{I}), \%\end{array}$ & $\begin{array}{l}\text { PCP } \\
(N=101), \%\end{array}$ & $\begin{array}{l}\text { NP/PA } \\
(\mathrm{N}=100), \%\end{array}$ & \\
\hline \multicolumn{6}{|c|}{ Characteristics ranked as top 3 that make a patient a good candidate } \\
\hline & Good kidney function & 36.6 & 29.7 & 42.0 & 0.191 \\
\hline & Good liver function & 3.0 & 4.0 & 9.0 & $0.120 *$ \\
\hline & No history of UTI & 19.8 & 9.9 & 13.0 & 0.120 \\
\hline & No history of GMI & 10.9 & 7.9 & 10.0 & 0.763 \\
\hline & Overweight or have weight concerns & 47.5 & 48.5 & 47.0 & 0.976 \\
\hline & Blood pressure & 13.9 & 12.9 & 10.0 & 0.687 \\
\hline & History of hypoglycemia & 19.8 & 24.8 & 16.0 & 0.301 \\
\hline & Been on other T2DM treatments for a long time & 18.8 & 16.8 & 13.0 & 0.525 \\
\hline & $\begin{array}{l}\text { Do not want an injectable/trying to delay injectables } \\
\text { as long as possible }\end{array}$ & 36.6 & 35.6 & 38.0 & 0.941 \\
\hline & Insurance coverage/affordability for long-term use & 38.6 & 44.6 & 42.0 & 0.692 \\
\hline & $\begin{array}{l}\text { Willing to take a medication that is "new" to } \\
\text { market }\end{array}$ & 7.9 & 11.9 & 10.0 & 0.642 \\
\hline & Willing to accept or put up with frequent urination & $12.9^{\mathrm{a}}$ & $22.8^{\mathrm{a}}$ & $13.0^{\mathrm{a}}$ & 0.091 \\
\hline & Overall treatment safety & 31.7 & 29.7 & 37.0 & 0.523 \\
\hline & Other & 2.0 & 1.0 & 0.0 & $1.000 *$ \\
\hline \multicolumn{6}{|c|}{ The treatment discussion and treatment decision maker } \\
\hline Provider makes the final & Never & $2^{\mathrm{a}}$ & $2^{\mathrm{a}}$ & 0.0 & $0.010^{*}$ \\
\hline \multirow[t]{2}{*}{ treatment decision } & Rarely/sometimes & $12.9^{\mathrm{a}}$ & $12.9^{\mathrm{a}}$ & $28.3^{b}$ & \\
\hline & Often/always & $85.1^{\mathrm{a}}$ & $85.1^{\mathrm{a}}$ & $71.7^{\mathrm{b}}$ & \\
\hline Provider discusses & Never & 1.0 & 2.0 & 1.0 & $0.897^{*}$ \\
\hline treatment and then & Rarely/sometimes & 9.9 & 6.9 & 7.1 & \\
\hline makes recommendation & Often/always & 89.1 & 91.1 & 91.9 & \\
\hline Provider discusses & Never & 3.0 & 2.0 & 1.0 & $0.950^{*}$ \\
\hline treatment and patient & Rarely/sometimes & 50.5 & 49.5 & 51.5 & \\
\hline makes decision & Often/always & 46.5 & 48.5 & 47.5 & \\
\hline \multicolumn{6}{|c|}{ Attributes of canagliflozin/canagliflozin-metformin typically discussed when discussing as a treatment option } \\
\hline Attributes typically & Mechanism of action & 67.7 & 59.0 & 65.0 & 0.426 \\
\hline \multirow[t]{15}{*}{ discussed } & Copay cards offered by manufacturer & $49.5^{\mathrm{a}, \mathrm{b}}$ & $40^{\mathrm{a}}$ & $60^{\mathrm{b}}$ & 0.018 \\
\hline & Dosing and administration & $59.6^{\mathrm{a}}$ & $53^{\mathrm{a}}$ & $73^{\mathrm{b}}$ & 0.012 \\
\hline & Urge/need for frequent urination & $54.5^{\mathrm{a}}$ & $37^{\mathrm{b}}$ & $55^{\mathrm{a}}$ & 0.015 \\
\hline & Out-of-pocket cost & 41.4 & 49.0 & 56.0 & 0.120 \\
\hline & Insurance coverage & 47.5 & 54.0 & 59.0 & 0.263 \\
\hline & Concomitant medication use & 29.3 & 26.0 & 39.0 & 0.120 \\
\hline & $\mathrm{HbAlc}$ reduction & 75.8 & 81.0 & 79.0 & 0.661 \\
\hline & Impact on weight & $81.8^{\mathrm{a}}$ & $63^{b}$ & $77^{a}$ & 0.007 \\
\hline & Lowering of systolic blood pressure & $58.6^{\mathrm{a}}$ & $44^{\mathrm{b}}$ & $44^{\mathrm{b}}$ & 0.060 \\
\hline & Potential rise in low-density lipoprotein (LDL) & 26.3 & 16.0 & 24.0 & 0.185 \\
\hline & cholesterol & & & & \\
\hline & UTI and/or GMI & $77.8^{\mathrm{a}}$ & $59.0^{\mathrm{b}}$ & $67.0^{\mathrm{a}, \mathrm{b}}$ & 0.017 \\
\hline & Risk of hypoglycemia & $54.5^{\mathrm{a}}$ & $36.0^{\mathrm{b}}$ & $57.0^{\mathrm{a}}$ & 0.005 \\
\hline & Overall treatment safety & $47.5^{\mathrm{a}, \mathrm{b}}$ & $38.0^{\mathrm{a}}$ & $56.0^{\mathrm{b}}$ & 0.039 \\
\hline & Other & 3.0 & 1.0 & 3.0 & $0.64 I^{*}$ \\
\hline
\end{tabular}

Note: *Test of independence conducted using Fisher's exact test when counts are $<5$. For pairwise comparisons, refer to superscripts (e.g., a, b, c) attached to column percentages. Column percentages in the same row that do not share the same superscripts (e.g., $X X^{a}$ vs $\left.X X^{b}\right)$ are significantly different at $p<0.05$. If the values share the same superscripts (e.g., $X X^{\mathrm{a}}$ vs $X X^{\mathrm{a}}$ or $X X^{\mathrm{a}, \mathrm{b}}$ vs $X X^{\mathrm{a}}$ ) then they are not significantly different at $p<0.05$. Only includes respondents who indicated that canagliflozin/canagliflozinmetformin was included in treatment discussion: endocrinologist $(n=99), \operatorname{PCP}(n=100), \operatorname{NP/PA}(n=100)$.

Abbreviations: PCP, primary care physician; NP/PA, nurse practitioners/physician assistants; UTI, urinary tract infection; GMI, genital mycotic infection; T2DM, type 2 diabetes mellitus; HbAlc, glycosylated hemoglobin.

Indeed, providers viewed canagliflozin as more favorable than DPP-4s for its effect on blood pressure and body weight and more favorable than SUs for its effect on $\mathrm{HbAlc}$, blood pressure, body weight, risk of hypoglycemia, and patient satisfaction. These findings are consistent with recent controlled trials which have shown that canagliflozin significantly reduces $\mathrm{HbA1c}$, body weight, and systolic blood pressure. In particular, head-to-head trials have shown canagliflozin 
Table 6 Positive changes observed by provider type (categorical) when managing patients with T2DM on canagliflozin/canagliflozinmetformin

\begin{tabular}{|c|c|c|c|}
\hline \multirow[t]{2}{*}{ Outcomes } & \multicolumn{3}{|l|}{ Provider type } \\
\hline & $\begin{array}{l}\text { Endocrinologist } \\
(\mathrm{N}=101), \%\end{array}$ & $\begin{array}{l}P C P \\
(N=101), \%\end{array}$ & $\begin{array}{l}\text { NP/PA } \\
(N=100), \%\end{array}$ \\
\hline \multicolumn{4}{|c|}{ Lowering/controlling $\mathrm{HbAlc}$} \\
\hline Never & 0.0 & 0.0 & 1.0 \\
\hline Rarely & 1.0 & 0.0 & 0.0 \\
\hline Sometimes & 14.9 & 17.8 & 11.0 \\
\hline Often/always & 84.2 & 82.2 & 88.0 \\
\hline \multicolumn{4}{|c|}{ Lowering/controlling blood pressure } \\
\hline Rarely & 7.9 & 6.9 & 6.0 \\
\hline Sometimes & 43.6 & 45.5 & 43.0 \\
\hline Often/always & 48.5 & 47.5 & 51.0 \\
\hline \multicolumn{4}{|l|}{ Weight loss } \\
\hline Never & 0.0 & 0.0 & 1.0 \\
\hline Rarely & 4.0 & 6.9 & 3.0 \\
\hline Sometimes & 27.7 & 33.7 & 43.0 \\
\hline Often/always & 68.3 & 59.4 & 53.0 \\
\hline \multicolumn{4}{|c|}{ Improvement in overall quality of life } \\
\hline Never & 1.0 & 0.0 & 0.0 \\
\hline Rarely & 6.9 & 7.9 & 5.0 \\
\hline Sometimes & 39.6 & 41.6 & 45.0 \\
\hline Often/always & 52.5 & 50.5 & 50.0 \\
\hline \multicolumn{4}{|c|}{ Feeling emotionally better that their T2DM is being managed well } \\
\hline Never & 0.0 & 1.0 & 0.0 \\
\hline Rarely & 5.0 & 6.9 & 6.0 \\
\hline Sometimes & 46.5 & 34.7 & 33.0 \\
\hline Often/always & 48.5 & 57.4 & 61.0 \\
\hline \multicolumn{4}{|c|}{ Behaving positively by adhering to treatment and/or follow-up visits } \\
\hline Never & 1.0 & 1.0 & 0.0 \\
\hline Rarely & 5.9 & 5.0 & 4.0 \\
\hline Sometimes & 38.6 & 37.6 & 33.0 \\
\hline Often/always & 54.5 & 56.4 & 63.0 \\
\hline \multicolumn{4}{|c|}{ Behaving positively by making changes to diet and exercise } \\
\hline Never & 1.0 & 0.0 & 0.0 \\
\hline Rarely & 9.9 & 9.9 & 9.0 \\
\hline Sometimes & 39.6 & 41.6 & 43.0 \\
\hline Often/always & 49.5 & 48.5 & 48.0 \\
\hline \multicolumn{4}{|c|}{ Having a hopeful outlook for managing T2DM } \\
\hline Never & 1.0 & 0.0 & 0.0 \\
\hline Rarely & 3.0 & 2.0 & 6.0 \\
\hline Sometimes & 41.6 & 40.6 & 37.0 \\
\hline Often/always & 54.5 & 57.4 & 57.0 \\
\hline
\end{tabular}

Note: There were no statistically significant differences across provider type.

Abbreviations: PCP, primary care physician; NP/PA, nurse practitioners/physician assistants; T2DM, type 2 diabetes mellitus; HbAIc, glycosylated hemoglobin.

to be more efficacious than sitagliptin and SUs in improving these outcomes. ${ }^{7,10,17-19}$

Real-world observational studies further corroborate these clinical studies, suggesting that canagliflozin is effective in reducing $\mathrm{HbAl} \mathrm{c}$ and lowering body weight. ${ }^{20}$ Notably, Thayer et al found that patients treated with canagliflozin were substantially more likely to reach the $<7 \% \mathrm{HbA} 1 \mathrm{c}$ target than patients treated with DPP-4 inhibitors. ${ }^{12}$ Indeed, the current study found that the attributes providers most frequently discussed with patients (when discussing canagliflozin) were $\mathrm{HbA1c}$ reduction and weight loss.

Health care providers reported positive experiences among patients using canagliflozin including improvement in overall QoL and having a more positive outlook and confidence in the management of their T2DM. Health care providers also observed improvement in patient behavioral outcomes including adherence to treatment and/or followup visits and changes to diet and exercise with the use of 
canagliflozin, which may have additional beneficial effects on clinical outcomes. Supporting these results, Buysman et al found that within a cohort of patients using canagliflozin, $86 \%$ were adherent to treatment over a 7-month period. ${ }^{14}$ Notably, although the current study did not assess adherence directly, but with physician recall of past patient behavior, it found similar results. Moreover, in clinical trials, canagliflozin use has been found to increase weight-related QoL and patient satisfaction. ${ }^{21}$ The weight loss associated with canagliflozin may act as positive feedback and lead to improvements in self-management behaviors including diet and exercise, which, in turn, leads to further weight loss and improvement in other clinical outcomes. ${ }^{21}$

Health care providers reported confidence in the use of canagliflozin as a treatment option for patients with T2DM, especially for use after metformin. Confidence was high, even in first-line treatment, although it was greater in the third and fourth line. This is consistent with AACE and ADA guidelines, which advise metformin to be used as first-line monotherapy unless contraindicated. ${ }^{3,4}$

This study adds to the growing body of literature providing real-world canagliflozin management experiences from health care providers, consisting of physician specialists, nonspecialists, and NA/PAs. Overall, providers reported favorable glycemic control, hypoglycemic risk, and weight loss. Health care providers also reported confidence in prescribing, and an improved overall experience of their patients in the management of their diabetes, in their practice setting, using canagliflozin.

\section{Limitations}

Although the study relied on self-reported evidence, selfreport is the only way to measure health care provider perceptions and personal experiences with treatment. The survey was cross-sectional; and therefore, not amenable to causal conclusions that may be drawn from an experimental and/or longitudinal survey. Representativeness was limited to providers identified through a panel, and further limited to respondents with access and motivation to participate in online studies. The provider's perception and experience with canagliflozin, compared with other SGLT2 inhibitors, regarding effectiveness and safety was not assessed in this current research study. Additional research is considered necessary to evaluate whether the physician-reported differences in health outcomes in the current study can be replicated by directly assessing patient outcomes in clinical practice.

\section{Conclusion}

Despite its relatively short time on the market, health care providers have reported favorable experiences with canagliflozin and canagliflozin-metformin FDC therapy. Specifically, health care providers have witnessed improvements in multiple clinical outcomes, as well as improvements in patients' experiences and even patients' self-management behaviors, among patients using canagliflozin. These outcomes should be viewed in the context of the Triple Aim framework given provider perception of improvement in health status and quality of care, which could affect costs.

\section{Acknowledgments}

This study was sponsored by Janssen Scientific Affairs, LLC. Kantar Health received funding from Janssen for study design, data collection, data analysis, and for the development of this manuscript. Jan-Samuel Wagner provided editorial support for manuscript preparation as a paid consultant to Kantar Health. Ryan Liebert provided assistance on data analyses and is a full-time employee of Kantar Health.

\section{Author contributions}

All authors (SCB, NMF, SH, and JC) jointly designed the research study. NMF conducted data management. SCB, NMF, and JC participated in data analysis. All authors made substantial contributions to the development and critical revision of the manuscript and approved the final version prior to submission.

\section{Disclosure}

Susan C Bolge was an employee of Janssen Scientific Affairs, LLC, during the time this research study was conducted and is currently an employee of Janssen Global Services, LLC. Also, she is a stockholder of Johnson \& Johnson, the parent company of Janssen Scientific Affairs, LLC and Janssen Global Services, LLC. Natalia M Flores and Shu Huang were fulltime employees of Kantar Health during the time this research study was conducted for which Janssen Scientific Affairs, LLC, provided funding to Kantar Health for data collection, analysis, and publication support. Jennifer Cai was an employee of Janssen Scientific Affairs, LCC during the time the study was conducted and is a stockholder of Johnson \& Johnson, the parent company of Janssen Scientific Affairs, LLC.

\section{References}

1. Centers for Disease Control and Prevention. National Diabetes Statistics Report: Estimates of Diabetes and Its Burden in the United States. Atlanta, GA: U.S. Department of Health and Human Services; 2014. 
2. American Diabetes Association. Economic costs of diabetes in the U.S. in 2012. Diabetes Care. 2013;36(4):1033-1046.

3. Chamberlain JJ, Rhinehart AS, Shaefer CF Jr, Neuman A. Diagnosis and management of diabetes: synopsis of the 2016 American Diabetes Association standards of medical care in diabetes. Ann Intern Med. 2016;164(8): $542-552$.

4. Garber AJ, Abrahamson MJ, Barzilay JI, et al. AACE/ACE comprehensive diabetes management algorithm 2015. Endocr Pract. 2015;21(4):438-447.

5. Bennett WL, Wilson LM, Bolen S, Maruthur N, et al; Agency for Healthcare Research and Quality. Oral diabetes medications for adults with type 2 diabetes: an update. 2011. Report No.: 11-EHC038-EF.

6. INVOKANA ${ }^{\circledR}$ (Canagliflozin) [Package Insert]. Titusville, NJ: Janssen Pharmaceuticals, Inc; 2015.

7. Stenlöf K, Cefalu WT, Kim KA, et al. Efficacy and safety of canagliflozin monotherapy in subjects with type 2 diabetes mellitus inadequately controlled with diet and exercise. Diabetes Obes Metab. 2013;15(4): 372-382.

8. Polidori D, Sha S, Mudaliar S, et al. Canagliflozin lowers postprandial glucose and insulin by delaying intestinal glucose absorption in addition to increasing urinary glucose excretion: results of a randomized, placebo-controlled study. Diabetes Care. 2013;36(8):2154-2161.

9. Cefalu W, Leiter L, Yoon K, et al. Efficacy and safety of canagliflozin versus glimepiride in patients with type 2 diabetes inadequately controlled with metformin (CANTATA-SU): 52 week results from a randomised, double-blind, phase 3 non-inferiority trial. Lancet. 2013;382(9896):941-950.

10. Schernthaner G, Gross J, Rosenstock J, et al. Canagliflozin compared with sitagliptin for patients with type 2 diabetes who do not have adequate glycemic control with metformin plus sulfonylurea. Diabetes Care. 2013;36(9):2508-2515.

11. Lefebvre P, Pilon D, Robitaille M-N, et al. Real-world glycemic, blood pressure, and weight control in patients with type 2 diabetes mellitus treated with canagliflozin-an electronic health-record-based study. Curr Med Res Opin Res Opin. 2016;32(6):1151-1159.
12. Thayer S, Chow W, Korrer S, Aguilar R. Real-world evaluation of glycemic control among patients with type 2 diabetes mellitus treated with canagliflozin versus dipeptidyl peptidase-4 inhibitors. Curr Med Res Opin. 2016;32(6):1087-1096.

13. Chow W, Buysman E, Rupnow MFT, Aguilar R, Henk HJ. Canagliflozin treatment of Hispanic and non-Hispanic patients with type 2 diabetes in a US managed care setting. Curr Med Res Opin. 2016;32(1): $13-22$.

14. Buysman EK, Chow W, Henk HJ, Rupnow MFT. Characteristics and outcomes of patients with type 2 diabetes mellitus treated with canagliflozin: a real-world analysis. BMC Endocr Disord. 2015;15(1):67.

15. Nisly SA, Kolanczyk DM, Walton AM. Canagliflozin, a new sodiumglucose cotransporter 2 inhibitor, in the treatment of diabetes. $\mathrm{Am} J$ Heal Pharm. 2013;70(4):311-319.

16. Berwick DM, Nolan TW, Whittington J. The triple aim: care, health, and cost. Health Aff. 2008;27(3):759-769.

17. Battise D. Efficacy and safety of canagliflozin as add-on therapy to metformin in type 2 diabetes. Clin Diabetes. 2014;32(2):81-86.

18. Leiter L, Yoon K, Arias P, et al. anagliflozin provides durable glycemic improvements and body weight reduction over 104 weeks vs glimepiride in patients with type 2 diabetes on metformin: a randomized, double-blind, phase 3 study. Diabetes Care. 2015;38(3): $355-364$.

19. Lavalle-González FJ, Januszewicz A, Davidson J, et al. Efficacy and safety of canagliflozin compared with placebo and sitagliptin in patients with type 2 diabetes on background metformin monotherapy: a randomised trial. Diabetologia. 2013;56(12):2582-2592.

20. Nardolillo A, Kane MP, Busch RS, Watsky J, Hamilton RA. A clinical perspective of canagliflozin in the management of type 2 diabetes mellitus. Clin Med Insights Endocrinol Diabetes. 2014;7:25-30.

21. Traina S, Guthrie R, Slee A. The impact of weight loss on weight-related quality of life and health satisfaction: results from a trial comparing canagliflozin with sitagliptin in triple therapy among people with type 2 diabetes. Postgrad Med. 2014;126(3):7-15. 


\section{Supplementary materials}

Table SI Patient characteristics by provider type

\begin{tabular}{|c|c|c|c|c|c|c|c|}
\hline \multirow[t]{3}{*}{ Patient characteristics } & \multicolumn{6}{|c|}{ Provider type } & \multirow{3}{*}{$\begin{array}{l}\text { One-way ANOVA } \\
\text { p-value }\end{array}$} \\
\hline & \multicolumn{2}{|c|}{$\begin{array}{l}\text { Endocrinologist } \\
(\mathrm{N}=101)\end{array}$} & \multicolumn{2}{|c|}{$\begin{array}{l}P C P \\
(N=|0|)\end{array}$} & \multicolumn{2}{|c|}{$\begin{array}{l}\text { NP/PA } \\
(N=100)\end{array}$} & \\
\hline & Mean & SD & Mean & SD & Mean & SD & \\
\hline \multicolumn{8}{|l|}{ Age (\% of patients, years) } \\
\hline$<18$ & $2.46^{\mathrm{a}}$ & 4.27 & $2.98^{\mathrm{a}}$ & 5.29 & $3.00^{\mathrm{a}}$ & 4.38 & 0.644 \\
\hline $18-44$ & $21.83^{\mathrm{a}, \mathrm{b}}$ & 9.29 & $20.03^{a}$ & 12.2 & $23.60^{b}$ & 11.03 & 0.069 \\
\hline $45-64$ & $35.62^{\mathrm{a}}$ & 11.89 & $35.93^{\mathrm{a}}$ & 13.84 & $37.19^{a}$ & 12.28 & 0.652 \\
\hline $65-74$ & $25.0 I^{a}$ & 8.94 & $26.30^{\mathrm{a}}$ & 12.75 & $24.8 I^{a}$ & 13.3 & 0.625 \\
\hline$\geq 75$ & $15.08^{\mathrm{a}}$ & 8.13 & $14.76^{\mathrm{a}}$ & 11.04 & $1 \mathrm{I} .40^{\mathrm{b}}$ & 11.54 & 0.021 \\
\hline \multicolumn{8}{|l|}{ Ethnicity (\% of patients) } \\
\hline White & $60.4 I^{a}$ & 21.32 & $59.72^{\mathrm{a}}$ & 26.83 & $46.80^{\mathrm{b}}$ & 23.08 & $<0.001$ \\
\hline Black or African American & $16.93^{\mathrm{a}}$ & 11.81 & $|7.6|^{\mathrm{a}}$ & 14.82 & $24.17^{b}$ & 16.73 & 0.001 \\
\hline Hispanic or Latino & $12.89^{a}$ & 10.45 & $13.75^{\mathrm{a}}$ & 15.35 & $18.79^{b}$ & $15.8 \mid$ & 0.006 \\
\hline Asian & $7.0 \mathrm{I}^{\mathrm{a}}$ & 7.72 & $6.96^{\mathrm{a}}$ & 11.38 & $5.91^{\mathrm{a}}$ & 5.93 & 0.597 \\
\hline $\begin{array}{l}\text { Other (mixed racial background, } \\
\text { native American, etc.) }\end{array}$ & $2.76^{\mathrm{a}, \mathrm{b}}$ & 5.3 & $1.95^{\mathrm{a}}$ & 4.69 & $4.33^{\mathrm{b}}$ & 11.03 & 0.078 \\
\hline \multicolumn{8}{|l|}{ Insurance (\% of patients) } \\
\hline Commercial & $56.16^{a}$ & 18.43 & $52.34^{a}$ & 19.13 & $43.6 \mathrm{I}^{\mathrm{b}}$ & 20.2 & $<0.001$ \\
\hline Medicaid & $10.10^{\mathrm{a}}$ & 10.38 & $11.28^{\mathrm{a}}$ & 11.15 & $19.17^{b}$ & 18.29 & $<0.001$ \\
\hline Medicare & $29.92^{\mathrm{a}}$ & 13.69 & $29.76^{\mathrm{a}}$ & 15.77 & $32.76^{\mathrm{a}}$ & 14.18 & 0.262 \\
\hline Uninsured & $3.65^{\mathrm{a}}$ & 4.4 & $6.93^{b}$ & 8.96 & $6.78^{\mathrm{b}}$ & 6.82 & 0.001 \\
\hline Other & $1.36^{\mathrm{a}}$ & 10.09 & $0.11^{\mathrm{a}}$ & 0.65 & $0.78^{\mathrm{a}}$ & 6.57 & 0.445 \\
\hline \multicolumn{8}{|l|}{ HbAlc level (\% of patients) } \\
\hline$<7.0$ & $34.80^{\mathrm{a}}$ & 20.53 & $41.33^{\mathrm{b}}$ & 21.57 & $30.97^{a}$ & 19.45 & 0.002 \\
\hline 7.0-7.9 & $28.06^{\mathrm{a}}$ & $1 \mathrm{I} .57$ & $29.82^{\mathrm{a}, \mathrm{b}}$ & 12.63 & $31.60^{\mathrm{b}}$ & $|2.7|$ & 0.127 \\
\hline $8.0-9.0$ & $22.15^{\mathrm{a}}$ & 12.37 & $18.04^{\mathrm{b}}$ & II & $23.06^{\mathrm{a}}$ & 11.24 & 0.005 \\
\hline \multirow[t]{2}{*}{$>9.0$} & $14.99^{a}$ & 8.92 & $10.8 \mathrm{I}^{\mathrm{b}}$ & 8.01 & $14.37^{\mathrm{a}}$ & 9.11 & 0.001 \\
\hline & $\mathbf{N}$ & $\%$ & $\mathbf{N}$ & $\%$ & $\mathbf{N}$ & $\%$ & Chi-square $p$-value \\
\hline \multicolumn{8}{|l|}{ Patient's education level } \\
\hline High School or less & $35^{\mathrm{a}}$ & 34.70 & $37^{\mathrm{a}, \mathrm{b}}$ & 36.60 & $49^{b}$ & 49.00 & 0.083 \\
\hline College or more & $4 I^{\mathrm{a}}$ & 40.60 & $40^{\mathrm{a}}$ & 39.60 & $24^{b}$ & 24.00 & \\
\hline Do not know & $25^{\mathrm{a}}$ & 24.80 & $24^{a}$ & 23.80 & $27^{\mathrm{a}}$ & 27.00 & \\
\hline
\end{tabular}

Notes: For pairwise comparisons, refer to superscripts (e.g., a, b, c) attached to column means. Column means in the same row that do not share the same superscripts (e.g., $X X^{a}$ vs $\left.X X^{b}\right)$ are significantly different at $p<0.05$. If the values share the same superscripts (e.g., $X X^{a}$ vs $X X^{a}$ or $X X^{a, b}$ vs $\left.X X^{a}\right)$ then they are not significantly different at $p<0.05$. Abbreviations: PCP, primary care physician; NP/PA, nurse practitioners/physician assistants; ANOVA, analysis of variance; HbAIc, glycosylated hemoglobin.

International Journal of General Medicine

\section{Publish your work in this journal}

The International Journal of General Medicine is an international, peer-reviewed open-access journal that focuses on general and internal medicine, pathogenesis, epidemiology, diagnosis, monitoring and treatment protocols. The journal is characterized by the rapid reporting of reviews, original research and clinical studies across all disease areas.

\section{Dovepress}

The manuscript management system is completely online and includes a very quick and fair peer-review system, which is all easy to use. Visit http://www.dovepress.com/testimonials.php to read real quotes from published authors. 